\title{
Non-radical, stepwise complete endoscopic resection of Barrett's epithelium in short segment Barrett's esophagus has a low stricture rate
}

Authors

Institutions
Andreas Koutsoumpas ${ }^{1}$, Lai Mun Wang ${ }^{2}$, Adam A. Bailey ${ }^{1}$, Richard Gillies ${ }^{3}$, Robert Marshall ${ }^{3}$, Michael Booth ${ }^{4}$, Bruno Sgromo $^{3}$, Nick Maynard ${ }^{3}$, Barbara Braden ${ }^{1}$

${ }^{1}$ Translational Gastroenterology Unit, Oxford University Hospitals, Oxford, UK

2 Department of Pathology, Oxford University Hospitals NHS Foundation Trust, Oxford, UK

${ }^{3}$ Department of Upper GI Surgery, Oxford University Hospitals, Oxford, UK

${ }^{4}$ Department of Surgery, Royal Berkshire Hospital, Reading, Berkshire, UK submitted 8. May 2016 accepted after revision 13. September 2016

\section{Bibliography}

Dol http://dx.doi.org/

10.1055/s-0042-118282

Published online: 2.12.2016

Endoscopy International Open 2016; 04: E1292-E1297

(c) Georg Thieme Verlag KG

Stuttgart · New York

E-ISSN 2196-9736

\section{Corresponding author}

\section{Professor Barbara Braden}

Consultant Gastroenterologist

Translational Gastroenterology Unit

Oxford University Hospitals

Oxford

OX3 9DU

UK

Fax: +44-1865-228763

braden@em.uni-frankfurt.de
Background and aims: Radical endoscopic excision of Barrett's epithelium performing 4-6 endoscopic resections during the same endoscopic session results in complete Barrett's eradication but has a high stricture rate (40-80\%). Therefore radiofrequency ablation is preferred after endoscopic mucosal resection (EMR) of visible nodules. We investigated the clinical outcome of non-radical, stepwise endoscopic mucosal resection with a maximum of two endoscopic resections per endoscopic session.

Methods: We analysed our prospectively maintained database of patients undergoing esophageal EMR for early neoplasia in Barrett's esophagus from 2009 to 2014. EMR was performed using a maximum of two band ligation mucosectomies per endoscopic session; thereafter, follow-up was 3-monthly and EMR was repeated as required for Barrett's eradication.

Results: In total, 118 patients underwent staging EMR for early Barrett's neoplasia. Subsequently, 27 patients underwent surgery/chemotherapy

\section{Introduction}

$\nabla$

During the last decades, endoscopic treatment has widely replaced esophagectomy as first choice therapy for early neoplasia related to Barrett's esophagus. The long-term outcome of endoscopic treatment and conventional esophagectomy for high grade dysplasia and intramucosal cancer is comparable but the adverse event rate and post-procedure quality of life are significantly in favor of endoscopic therapy.

Endoscopic resection of visible nodules followed by ablation of the remaining Barrett's epithelium and endoscopic surveillance is the currently recommended standard treatment for high grade dysplasia and intramucosal cancer in Barrett's esophagus [1-3]. Endoscopic resection followed by radiofrequency ablation can achieve complete remission of dysplasia in more than $90 \%$ [3-5] due to deep submucosal or more advanced tumor stages or were managed conservatively. The remaining 91 patients with high grade dysplasia (48), intramucosal (38) or submucosal cancer (5) in the resected nodule underwent further endoscopic therapy with a mean follow-up of 24 months. Remission of dysplasia/neoplasia was achieved in 95.6\% after 12 months treatment. Stepwise endoscopic Barrett's resection resulted in complete Barrett's eradication in 36/91 patients (39.6\%) in a mean of four sessions; 40/91 patients $(44.0 \%)$ had a short circumferential Barrett's segment $(<3 \mathrm{~cm})$. In this group, repeated EMR achieved complete Barrett's excision in $85.0 \%$. One patient developed a stricture $(1.1 \%)$, one a delayed bleeding, and there were no perforations.

Conclusion: In patients with a short Barrett's segment, non-radical endoscopic Barrett's resection at the time of scheduled endoscopy follow-up allows complete Barrett's eradication with very low stricture rate.

and complete remission of intestinal metaplasia in more than $77 \%$ of patients $[6,7]$.

Radiofrequency ablation allows the precise ablation of the columnar lined epithelium to a depth of about 500 to $700 \mu \mathrm{m}$, which usually comprises the mucosa and the upper parts of the submucosa. However, the disposable catheter probes for radiofrequency ablation are expensive and the costly generator equipment is not widely available. Moreover, radiofrequency ablation works by tissue destruction thus not providing histology; this might confer the small risk of burying an endoscopically unrecognized invasive cancer [8, 9].

Radical endoscopic resection for complete eradication of Barrett's epithelium was initially proposed as a definitive therapy but has not been pursued further due to a high stricture rate (48$88 \%$ ) when 4-5 resections were performed 
within the same endoscopic session $[4,6,10,11]$. However, the question remains whether a non-radical approach with stepwise endoscopic resection in more frequent endoscopic sessions would also achieve complete remission of intestinal metaplasia but could avoid such a high rate of adverse events.

In our retrospective study from a prospectively maintained database, we investigated the outcome of stepwise, non-radical endoscopic resection to achieve complete remission of dysplasia and complete remission of intestinal metaplasia using no more than two band ligation mucosectomies per session.

\section{Methods}

$\nabla$

\section{Patients}

Between May 2009 and December 2014, consecutive patients undergoing EMR for biopsy-proven high grade dysplasia (HGD) or early esophageal cancer in Barrett's esophagus were prospectively audited in a database and enrolled into this study. Endoscopic ultrasound was routinely carried out in all patients with visible nodules of more than $1 \mathrm{~cm}$ size. Patients with endosonographically detected infiltration of the muscularis propria or obvious lymph node involvement on EUS, CT or PET-CT were excluded. All patients eligible for esophageal endoscopic resection were discussed and agreed at the Upper Gastrointestinal Multidisciplinary Team (MDT) meeting.

The study adheres to the principles outlined in the Declaration of Helsinki. Informed consent was obtained from all patients. Patients were informed in detail about the risks and benefits of the endoscopic treatment and surgical and endoscopic alternatives. The observational nature of the study was established with the Health Research Authority and Trust R\&D department. The study was therefore registered locally in accordance with Trust clinical governance guidelines.

\section{Standard protocol for endoscopic mucosal resection (EMR) and follow-up}

After a fasting period of at least 4 hours, esophagogastroduodenoscopy was performed by an interventional endoscopist or a directly supervised clinical fellow using high definition white-light endoscopy (Olympus Evis Lucera processor, GIF H260 and GIF 1T140, Olympus, Tokyo, Japan) under conscious sedation with intravenous midazolam and fentanyl. The length of the Barrett's segment was reported according to the Prague classification [12], visible lesions were described based on the Paris endoscopic classification. The position of nodules was reported by distance from the incisors and using the clock face in neutral position of the endoscope.

Acetic acid staining (1.5 v/v\%) and narrow-band imaging (NBI) were routinely applied to improve the visualization and delineation of neoplastic lesions. Targeted biopsies and Seattle protocol biopsies were taken. Biopsies and EMR specimens were examined by two expert gastrointestinal histopathologists according to the Vienna classification system of esophageal neoplasia [13]. Endoscopic mucosal resection of visible nodules and for Barrett's eradication was performed using the band ligation technique (Duette Multiband Mucosectomy Kit, Cook Ireland Limited, Limerick, Ireland) and therapeutic endoscopes (Olympus). The endo$\mathrm{cut}^{\circledR}$ setting of the diathermy unit (ERBE VIO300D; ERBE Elektromedizin, Tübingen, Germany) was used for the endoscopic resection. A maximum of two band ligation resections was performed per endoscopic session and overlapping resections were avoided when possible. All patients were discharged the same day after 1 to 2 hours of observation. Patients were advised to continue proton pump inhibitors twice daily for the duration of endoscopic treatment.

The histology of the staging EMR was reviewed and discussed at the MDT and patients were considered for surgery if the resected specimen showed esophageal adenocarcinoma with positive deep resection margins or high risk features for locally advanced disease (submucosal infiltration, lymphovascular invasion or poor differentiation). Patients with high grade dysplasia or intramucosal adenocarcinoma with negative deep resection margins were considered suitable for further endoscopic therapy. Patients with only superficial infiltration of the submucosa $(<500 \mu \mathrm{m}$; T1b sm1) were offered surgery or endoscopic treatment after explaining the risk of lymph node infiltration of about $2-8 \%$ [1416].

After initial endoscopic resection of visible nodules, endoscopies with the option for further resections of the Barrett's epithelium aiming at complete remission of intestinal metaplasia were repeated 3-monthly for 1 year.

If no dysplasia was detected on biopsies, then the interval was extended to 6 months thereafter. When complete remission of dysplasia and intestinal metaplasia had been achieved, patients underwent annual endoscopic surveillance.

Patients with residual columnar lined epithelium after four sessions of endoscopic resection and follow-up of more than 12 months were considered for radiofrequency ablation unless they had severe comorbidities significantly reducing their life expectancy.

Argon plasma coagulation was only allowed for tiny Barrett's islands of less than $2 \mathrm{~mm}$ diameter.

The presence of residual Barrett's epithelium was assessed visually using NBI inspection and histologically by taking quadrantic biopsies.

\section{Adverse events}

When attending the follow-up endoscopies, patients were routinely asked whether adverse events such as hematemesis, melena, and dysphagia had occurred since the last endoscopy.

Dysphagia was assessed using a validated dysphagia score -0 : able to eat conventional diet, 1 : able to eat some solid meal, 2 : able to eat semisolid but not solid food, 3: able to drink fluids only, 4: aphagia [17].

Strictures were defined as dysphagia score $\geq 1$ or the need for endoscopic dilatation.

\section{Outcome parameters}

Study end points were:

(1) The rate of complete remission of intestinal metaplasia, high grade dysplasia and malignancy post completion of endoscopic therapy as well as the rate of complete eradication of the Barrett's epithelium after a follow-up of at least 1 year. Complete Barrett's eradication was defined as no visible Barrett's epithelium using narrow-band imaging (COMO Prague classification).

(2) Adverse events such as bleeding, perforation or stricture rate were evaluated.

\section{Statistics}

Variables analysed included patient characteristics, histological results, and frequency of complications. Values are presented as medians with $25 \%$ to $75 \%$ interquartile range (IQR), and $95 \%$ con- 


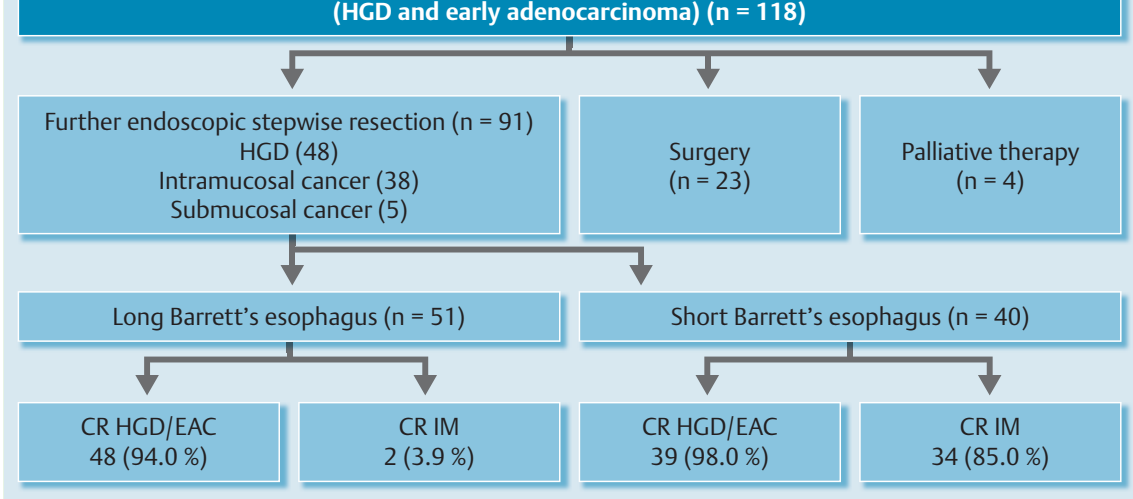

Fig. 1 Flowchart for patients.

Short Barrett's esophagus ( $C<3 \mathrm{~cm}$ and $\mathrm{M}<5 \mathrm{~cm}$ ).

Long Barrett's esophagus ( $C>3 \mathrm{~cm}$ and $/$ or $M>5 \mathrm{~cm}$ ).

HGD - High grade dysplasia.

CR IM - Complete remission of intestinal metaplasia.

CR HGD/EAC - Complete remission of high grade dysplasia and early adenocarcinoma.

fidence intervals (CIs). $P$ values of less than 0.05 were considered statistically significant,

\section{Results}

\section{$\nabla$}

During the study period, 118 patients underwent staging EMR for early neoplasia in Barrett's epithelium. Subsequently, 23 patients underwent surgery/chemotherapy due to deep submucosal or more advanced tumor stages (infiltration of the submucosa with positive deep margins), and four patients were managed conservatively due to patient fitness, comorbidities, or patient choice. The flowchart for patients is illustrated in $\bullet$ Fig. 1.

After reviewing the histology of the resected esophageal lesion and discussing the clinical context, 91 patients were considered suitable for further endoscopic therapy. Among the patients undergoing further endoscopic treatment, 48 patients had high grade dysplasia, 38 intramucosal cancer, and five submucosal cancer (T1sm1 defined as submucosal infiltration depth of less than $500 \mu \mathrm{m}$ ) in the endoscopically resected specimen at index endoscopy. The characteristics of the 91 patients receiving further endoscopic treatment are given in Table1. Median follow-up of the endoscopically treated patients was 30 months (IQR 16-52 months). Complete remission of HGD/neoplasia was achieved in $95.6 \%$ of all endoscopically treated patients after 12 months. In four patients, high grade dysplasia or cancer was still present after 12 months: one patient with submucosal adenocarcinoma in the initial EMR specimen but unfit for surgery developed local lymphadenopathy and was treated with chemoradiotherapy. One patient with multifocal high grade dysplasia in a long Barrett's segment suffered a hemorrhagic stroke and further endoscopic treatment was subsided. In a patient with completely resected short Barrett's segment, a focus of intramucosal cancer was found in a residual $3 \mathrm{~mm}$ Barrett's island after 12 months and completely resected endoscopically. A fourth patient with intramucosal cancer and multifocal high grade dysplasia in a long Barrett's segment still had focal high grade dysplasia on biopsies. He underwent radiofrequency ablation after endoscopic resection of all visible nodules.

Stepwise endoscopic Barrett's resection resulted in complete Barrett's eradication in 36 patients (39.6\%) in a mean of four sessions.

From the patients with remaining Barrett's epithelium after 1 year, 31 patients with long segment Barrett's esophagus subsequently underwent radiofrequency ablation.

\section{Short Barrett's esophagus}

In total, 40 patients (44.0\%) had a short circumferential Barrett's segment $(\mathrm{C}<3 \mathrm{~cm}, \mathrm{M}<5 \mathrm{~cm}$ according to the Prague classification); the median circumferential length was $1 \mathrm{~cm}$ (IQR $1-2 \mathrm{~cm})$, and the median maximal length of columnar lined epithelium was $3 \mathrm{~cm}(2-3 \mathrm{~cm})$.

In this group, repeated EMR achieved complete remission of intestinal metaplasia in $85.0 \%$ in a median of four EMR sessions (IQR 1 - 5). In one patient who had high grade dysplasia in the initial staging EMR, an intramucosal cancer was detected in a $3 \mathrm{~mm}$ Barrett's island after 12 months follow-up (metachronous cancer) and this was subsequently completely resected. Complete remission of high grade dysplasia and early cancer was accom-

\begin{tabular}{|c|c|}
\hline Sex, M/F & $73 / 18(80.2 \% \mid 19.8 \%)$ \\
\hline Mean age (SD), years & $71(11)$ \\
\hline Median circumferential length ( $C$ in Prague classification) (IQR), $\mathrm{cm}$ & $4(1-6)$ \\
\hline Median maximal length (M in Prague classification) (IQR), cm & $6(3-8)$ \\
\hline Short Barrett's esophagus $(C<3 \mathrm{~cm}$ and $M<5 \mathrm{~cm}), \mathrm{n}(\%)$ & $40(43.9)$ \\
\hline \multicolumn{2}{|l|}{ Histology } \\
\hline HGD, n (\%) & $48(52.7)$ \\
\hline Intramucosal cancer, $\mathrm{n}(\%)$ & $38(41.8)$ \\
\hline Submucosal cancer, $\mathrm{n}(\%)$ & $5(5.5)$ \\
\hline Median follow-up (IQR), months & $30(16-52)$ \\
\hline
\end{tabular}

Table 1 Characteristics of patients selected for stepwise endoscopic resection of high grade dysplasia and early esophageal cancer in Barrett's esophagus $(n=91)$. 


\begin{tabular}{|c|c|c|c|}
\hline Authors & $\mathbf{n}$ & $\begin{array}{l}\text { Complete remission of } \\
\text { dysplasia, \% }\end{array}$ & $\begin{array}{l}\text { Complete remission of } \\
\text { intestinal metaplasia, \% }\end{array}$ \\
\hline Shaheen et al. (2009) [3] & 127 & 90 & 77 \\
\hline Pouw et al. (2010) [6] & 52 & 100 & 96 \\
\hline Alvarez Herrero et al. (2011) [19] & 26 & 83 & 79 \\
\hline Van Vilsteren et al. (2011) [4] & 22 & 96 & 96 \\
\hline Kim et al. (2012) [5] & 65 & 94 & 88 \\
\hline Bulsiewicz et al. (2013) [7] & 244 & 87 & 80 \\
\hline Haidry et al. (2015) [2] & 515 & 88 & 76 \\
\hline
\end{tabular}

Table 2 Outcome of endoscopic resection followed by radiofrequency ablation in the literature.

\begin{tabular}{|c|c|c|c|}
\hline Authors & Patients & EMRs per session & Stricture rate, \% \\
\hline Oxford cohort (this study) & 91 & 2 & 1.1 \\
\hline Soehendra et al. (2006) [24] & 10 & 6 & 70 \\
\hline Van Vilsteren et al. (2011) [4] & 25 & 5 & 88 \\
\hline Pouw et al. (2010) [20] & 169 & 4 & 49 \\
\hline Chung et al. (2011) [21] & 77 & 3 & 33 \\
\hline Larghi et al. (2007) [22] & 26 & 3 & 12 \\
\hline Alvarez Herrero et al. (2011) [10] & 69 & 5 & 48 \\
\hline
\end{tabular}

Table 3 Stricture rate after endoscopic mucosal resection related to the number of resections per endoscopic session. plished in $98.0 \%$ of the subgroup of patients with short segment Barrett's esophagus.

In seven patients with short Barrett's esophagus, additional argon plasma coagulation was applied to ablate small Barrett's islands of less than $2 \mathrm{~mm}$.

\section{Adverse events}

All patients were discharged on the same day after endoscopic resection. One patient reported dysphagia to solids (dysphagia score: 1 ) and had developed a mild stricture (1.1\%) which was dilated with a balloon in one endoscopic session.

No perforations were observed in the total of 333 EMR sessions. Immediate bleeding requiring endoscopic intervention during the procedure occurred in $7.1 \%$ of patients. Intraprocedural bleeding was successfully stopped in all these patients by heater probe, hemoclips, adrenalin injection or soft-coagulation using the snare tip.

One patient with a long Barrett's segment who was taking warfarin for atrial fibrillation developed delayed bleeding 10 days after endoscopic resection and was readmitted with melena and a drop in hemoglobin of $20 \mathrm{~g} / \mathrm{L}$. The bleeding from a resection ulcer had already stopped spontaneously at the time of endoscopy.

\section{Discussion}

The incidence of esophageal adenocarcinoma is increasing. Due to advanced endoscopic imaging techniques and improved surveillance programs for Barrett's esophagus, detection of early esophageal neoplasia such as high grade dysplasia and intramucosal cancer has become more frequent. The recommended standard treatment for such early esophageal neoplastic alterations in Barrett's epithelium is endoscopic resection of visible lesions followed by radiofrequency ablation of the columnar lined epithelium. Ablation of the residual Barrett's esophagus is advised due to the high risk of metachronous cancer of about $20 \%$ $[1,18]$. Using these combined endoscopic treatment modalities, complete remission of dysplasia is obtained in $83-100 \%$ and complete remission of intestinal metaplasia in $76-96 \%$ of patients ( Table 2 ).
In contrast to radiofrequency ablation which is a tissue destroying ablation method, endoscopic resection for Barrett's ablation provides the important advantage of histological assessment and more accurate staging. It can also detect occult synchronous cancer which might have been missed despite careful endoscopic inspection with advanced imaging methods.

Previous studies have reported high stricture rates for radical endoscopic resection of Barrett's epithelium; when four or five mucosal resections were performed in the same endoscopic session, the stricture rate was unacceptably high at $48-88 \%$ [4, 19,20] ( Table 2; [2-7,19]). Performing only three resections per session showed lower stricture rates of $33 \%$ [21,22]. If complete endoscopic resection of a short Barrett's segment $(\mathrm{C}<3 \mathrm{~cm}$, $\mathrm{M}<5 \mathrm{~cm}$ ) is achieved in a radical approach within a median of two EMR sessions, the proportion of patients requiring dilatation of a stricture was $36.8 \%$ [23].

In our cohort, the stricture rate after non-radical stepwise endoscopic resection was very low $(1 / 91 ; 1.1 \%)$ and multiple dilatations were not required. We did not observe a high complication rate as reported for radical complete endoscopic resection in other studies ( Table 3; [4,10,20-24]), and we explain our favorable outcome by the low number of resections per endoscopic session; a maximum of two band ligation resections were performed and we avoided overlapping resections when possible. If overlapping resections were required, we tended to resect more proximal or distal to the mucosal defect rather than horizontally at the same distance from the incisors. None of the resections affected more than the semi-circumference. This means that the mucosal defect in regard to the circumference was relatively small which seems to reduce the risk of stricture development. It is known that the stricture risk is highest when circumferential resection is performed. Qumseya et al. identified the number of resections in the index procedure and the size of the lesions removed as risk factors in developing a stricture [25].

Other EMR studies have followed intensified protocols, offering shortened intervals for repeat EMR at 6-8 weeks [23]. Although the optimum interval for a repeat procedure is unclear, we find that repeating 3-monthly gastroscopies/EMRs is sufficient, since it does not lead to an increased rate of metachronous cancers in short segment Barrett's and allows the ligation ulcers to heal. However, careful inspection of the Barrett's epithelium, prefer- 
ably using narrow-band imaging and/or acetic acid chromoendoscopy is mandatory at each endoscopic session to minimize the risk of leaving neoplastic tissue behind for a further 3 months. Obviously, a less aggressive protocol which reflects the conventional follow-up intervals reduces inconvenience to the patient and the endoscopy unit's burden.

Endoscopic mucosal resection using the band ligation technique has a favorable safety profile as also demonstrated in our study. In large cohorts, major events such as bleeding or perforation are very uncommon. As the stricture rate is very low when using only two band ligations per session, further endoscopic resection is not hindered by stenosis in later resections. We also did not encounter fibrosis after previous endoscopic resections which could render the suction of the mucosa into the attached cap difficult during subsequent band ligations.

After radiofrequency ablation, the rate of strictures requiring endoscopic dilation is also substantial and is reported to be $6 \%$ in the American registry [26]. In the UK registry, an improvement in the stricture rate after radiofrequency ablation could be observed from $9.2 \%$ in the years $2008-2010$ to $6.2 \%$ between 2011 and 2013 [27].

Our findings support stepwise, non-radical endoscopic resection in short segment Barrett's esophagus as a safe, highly effective and less expensive alternative to radiofrequency ablation with an even lower stricture rate.

Within a year and up to eight endoscopic band ligation resections, $85 \%$ of the short Barrett's segments $(C<3 \mathrm{~cm})$ could be completely eradicated endoscopically in our study. The median diameter of the resected specimen was $18 \mathrm{~mm}$ giving an approximate mucosal area of $2.5 \mathrm{~cm}^{2}$ when assuming a circular shape $\left(\pi r^{2}\right)$. A typical $25 \mathrm{~mm}$ diameter Barrett's esophagus of longer than C3M3 would have an approximate surface area of $>23.4 \mathrm{~cm}^{2} \quad\left(2 \pi r \times 30 \mathrm{~mm}=78 \mathrm{~mm} \times 30 \mathrm{~mm}=23.4 \mathrm{~cm}^{2}\right)$. This implies that a long Barrett's esophagus would require more than nine band ligation resections to cover the area and explains why only two $(3.9 \%)$ of the patients with longer Barrett's segments achieved complete remission of intestinal metaplasia within four endoscopic sessions in our study.

To prevent stricture formation after extensive endoscopic resection, the use of oral steroids, the endoscopic injection of steroids or topical application of hemostatic powder or polyglycolic acid sheets have recently been suggested [28-32]. Although these techniques seem to be promising, none of the available preventive methods is efficient and safe enough to justify recommendation for routine use in clinical practice [33].

The main limitation of our single center/single operator study is its retrospective uncontrolled design; however, complications and outcome have been continuously audited in a prospectively maintained database since 2009. It must be borne in mind that we are a tertiary center practicing advanced endoscopic imaging and interventional procedures routinely, and our results may not be replicated in every other endoscopy unit setting. However, we believe that patients with early esophageal neoplasia should be treated in high volume expert centers with multidisciplinary teams and with expertise in advanced endoscopic imaging, endoscopic treatment, minimally invasive surgery, oncology, and pathology. Randomized controlled multicenter studies with direct comparison between radiofrequency ablation and non-radical stepwise endoscopic resection in short segment Barrett's esophagus are desirable.

To our knowledge, this is the first cohort of patients with short segment Barrett's esophagus treated with consecutive EMR re- sections, with a maximum of two resections per session. This less aggressive resection protocol that we propose has not been systemically evaluated in the past.

In conclusion, stepwise, non-radical endoscopic resection is a well-tolerated, safe and effective alternative to radiofrequency ablation in short segment Barrett's esophagus; it achieves complete remission of dysplasia and intestinal metaplasia with low complication rates. In contrast to a more radical approach with multiple resections in a single endoscopic session, the stepwise non-radical approach results in a low stricture rate.

\section{Competing interests: None}

\section{References}

1 Fitzgerald RC, di Pietro M, Ragunath K et al. British Society of Gastroenterology guidelines on the diagnosis and management of Barrett's oesophagus. Gut 2014; 63: 7-42

2 Haidry RJ, Lipman G, Banks MR et al. Comparing outcome of radiofrequency ablation in Barrett's with high grade dysplasia and intramucosal carcinoma: a prospective multicenter UK registry. Endoscopy 2015; 47: $980-987$

3 Shaheen NJ, Sharma P, Overholt BF et al. Radiofrequency ablation in Barrett's esophagus with dysplasia. NEJM 2009; 360: 2277-2288

4 van Vilsteren FGI, Pouw RE, Seewald S et al. Stepwise radical endoscopic resection versus radiofrequency ablation for Barrett's oesophagus with high-grade dysplasia or early cancer: a multicentre randomised trial. Gut 2011; 60: 765 - 773

5 Kim HP, Bulsiewicz WJ, Cotton CC et al. Focal endoscopic mucosal resection before radiofrequency ablation is equally effective and safe compared with radiofrequency ablation alone for the eradication of Barrett's esophagus with advanced neoplasia. Gastrointest Endosc 2012; 76: $733-739$

6 Pouw RE, Wirths $K$, Eisendrath $P$ et al. Efficacy of radiofrequency ablation combined with endoscopic resection for barrett's esophagus with early neoplasia. Clin Gastroenterol Hepatol 2010; 8: 23-29

7 Bulsiewicz WJ, Kim HP, Dellon ES et al. Safety and efficacy of endoscopic mucosal therapy with radiofrequency ablation for patients with neoplastic Barrett's esophagus. Clin Gastroenterol Hepatol 2013; 11: 636-642

8 Gray NA, Odze RD, Spechler SJ. Buried metaplasia after endoscopic ablation of Barrett's esophagus: a systematic review. Am J Gastroenterol 2011; 106: 1899-1908

9 Lee JK, Cameron RG, Binmoeller KF et al. Recurrence of subsquamous dysplasia and carcinoma after successful endoscopic and radiofrequency ablation therapy for dysplastic Barrett's esophagus. Endoscopy 2013; 45: 571-574

10 Alvarez Herrero L, Pouw RE, van Vilsteren FGI et al. Safety and efficacy of multiband mucosectomy in 1060 resections in Barrett's esophagus. Endoscopy 2011; 43: 177-183

11 Pouw RE, Peters FP, Sempoux C et al. Stepwise radical endoscopic resection for Barrett's esophagus with early neoplasia: report on a Brussels' cohort. Endoscopy 2008; 40: 892-898

12 Sharma P, Dent J, Armstrong $D$ et al. The development and validation of an endoscopic grading system for Barrett's esophagus: the Prague C \& M criteria. Gastroenterology 2006; 131: 1392-1399

13 Schlemper RJ, Riddell RH, Kato $Y$ et al. The Vienna classification of gastrointestinal epithelial neoplasia. Gut 2000; 47: 251-255

14 Manner $H$, Pech $O$, Heldmann $Y$ et al. The frequency of lymph node metastasis in early-stage adenocarcinoma of the esophagus with incipient submucosal invasion (pT1b sm1) depending on histological risk patterns. Surg Endosc 2015; 29: 1888-1896

15 Ancona E, Rampado S, Cassaro $M$ et al. Prediction of lymph node status in superficial esophageal carcinoma. Ann Surg Oncol 2008; 15: 3278 3288

16 Liu L, Hofstetter WL, Rashid A et al. Significance of the depth of tumor invasion and lymph node metastasis in superficially invasive (T1) esophageal adenocarcinoma. Am J Surg Pathol 2005; 29: 1079-1085

17 Knyrim K, Wagner HJ, Bethge $N$ et al. A controlled trial of an expansile metal stent for palliation of esophageal obstruction due to inoperable cancer. NEJM 1993; 329: $1302-1307$

18 Pech O, Behrens A, May A et al. Long-term results and risk factor analysis for recurrence after curative endoscopic therapy in 349 patients 
with high-grade intraepithelial neoplasia and mucosal adenocarcinoma in Barrett's oesophagus. Gut 2008; 57: 1200-1206

19 Alvarez Herrero L, van Vilsteren FGI, Pouw RE et al. Endoscopic radiofrequency ablation combined with endoscopic resection for early neoplasia in Barrett's esophagus longer than $10 \mathrm{~cm}$. Gastrointest Endosc 2011; 73: $682-690$

20 Pouw RE, Seewald S, Gondrie JJ et al. Stepwise radical endoscopic resection for eradication of Barrett's oesophagus with early neoplasia in a cohort of 169 patients. Gut 2010; 59: 1169-1177

21 Chung A, Bourke MJ, Hourigan LF et al. Complete Barrett's excision by stepwise endoscopic resection in short-segment disease: long term outcomes and predictors of stricture. Endoscopy 2011; 43: 10251032

22 Larghi A, Lightdale CJ, Ross AS et al. Long-term follow-up of complete Barrett's eradication endoscopic mucosal resection (CBE-EMR) for the treatment of high grade dysplasia and intramucosal carcinoma. Endoscopy 2007; 39: $1086-1091$

23 Bahin FF, Jayanna M, Hourigan LF et al. Long-term outcomes of a primary complete endoscopic resection strategy for short-segment Barrett's esophagus with high-grade dysplasia and/or early esophageal adenocarcinoma. Gastrointest Endosc 2016; 83: 68 - 77

24 Soehendra N, Seewald S, Groth S et al. Use of modified multiband ligator facilitates circumferential EMR in Barrett's esophagus (with video). Gastrointest Endosc 2006; 63: 847-852

25 Qumseya B, Panossian AM, Rizk C et al. Predictors of esophageal stricture formation post endoscopic mucosal resection. Clin Endosc 2014; 47: $155-161$

26 Li N, Pasricha S, Bulsiewicz WJ et al. Effects of preceding endoscopic mucosal resection on the efficacy and safety of radiofrequency ablation for treatment of Barrett's esophagus: results from the United
States Radiofrequency Ablation Registry. Dis Esophagus 2016; 29: 537-543. DOI 10.1111/dote. 12386

27 Haidry RJ, Butt MA, Dunn JM et al. Improvement over time in outcomes for patients undergoing endoscopic therapy for Barrett's oesophagusrelated neoplasia: 6 -year experience from the first 500 patients treated in the UK patient registry. Gut 2015; 64: $1192-1199$

28 Hanaoka $N$, Ishihara $R$, Takeuchi $Y$ et al. Intralesional steroid injection to prevent stricture after endoscopic submucosal dissection for esophageal cancer: a controlled prospective study. Endoscopy 2012; 44: $1007-1011$

29 Hashimoto S, Kobayashi M, Takeuchi M et al. The efficacy of endoscopic triamcinolone injection for the prevention of esophageal stricture after endoscopic submucosal dissection. Gastrointest Endosc 2011; 74: $1389-1393$

30 Beye B, Barret M, Alatawi $A$ et al. Topical hemostatic powder promotes reepithelialization and reduces scar formation after extensive esophageal mucosal resection. Dis Esophagus 2016; 29: 520-527. DOI 10.1111/dote. 12378

31 Iizuka T, Kikuchi D, Yamada A et al. Polyglycolic acid sheet application to prevent esophageal stricture after endoscopic submucosal dissection for esophageal squamous cell carcinoma. Endoscopy 2015; 47: $341-344$

32 Bahin FF, Jayanna $M$, Williams SJ et al. Efficacy of viscous budesonide slurry for prevention of esophageal stricture formation after complete endoscopic mucosal resection of short-segment Barrett's neoplasia. Endoscopy 2016; 48: $71-74$

33 Barret $M$, Beye B, Leblanc $S$ et al. Systematic review: the prevention of oesophageal stricture after endoscopic resection. Aliment Pharmacol Ther 2015; 42: 20-39 